\title{
Traffic Image Classification using Horizontal Slice Algorithm
}

\author{
Luong Anh Tuan Nguyen \\ Faculty of InformationTechnology \\ HCMC University of Transport \\ No.2, D3 Street, Ward 25, \\ Binh Thanh District, \\ Ho Chi Minh City Vietnam
}

\author{
Thi-Ngoc-Thanh Nguyen \\ Faculty of InformationTechnology \\ HCMC University of Transport \\ No.2, D3 Street, Ward 25, \\ Binh Thanh District, \\ Ho Chi Minh City, Vietnam
}

\begin{abstract}
Traffic image classification to identify the traffic density will support the traffic problems such as intelligent traffic signal control, traffic planning, etc. This paper proposes a novel traffic image classification method based on horizontal slice algorithm and histogram. The system model is designed and evaluated with the image datasets of Ho Chi Minh city, Vietnam. The best accuracy result can obtain $91 \%$.
\end{abstract}

\section{General Terms}

Traffic image classification, horizontal slice algorithm, histogram

\section{Keywords}

Traffic image classification, horizontal slice algorithm, histogram

\section{INTRODUCTION}

Currently, there are several methods of identifying traffic density $[1,2]$, although the technique is effect but complicated calculations.

It is easy to find that the traffic image with crowded traffic density, the traffic object will appear most of positions in image. Besides, the objects in image have the contrast with the background, so it is easy to distinguish between objects and the background. This paper proposes a novel technique to classify traffic density based on horizontal slice algorithm and histogram.

The rest of the paper is organized as follows. The theoretical background is discussed in Section 2. Section 3 presents the design of system model. In section 4, the numerical results of experiment are illustrated. Finally, Section 5 concludes this paper and figures out the future works.

\section{THEORETICAL BACKGROUND}

\subsection{Monochrome Image}

A monochrome image $[3,4,5]$ is a 2-D array of pixels. Each element of array is a pixel. Each pixel occupies one byte of memory. The total number of bytes in the image is $\mathrm{MxN}$ bytes (M: rows, $\mathrm{N}$ : columns). The following equation illustrates the pixel value of monochrome image.

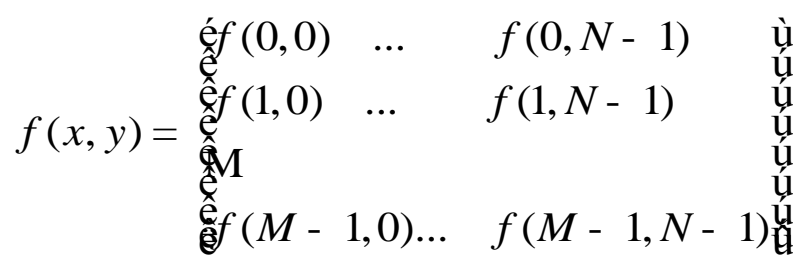

Where, $f(x, y) \in Z$ ( $\mathrm{Z}$ belongs to the positive integers), and 0 $=<\mathrm{f}(\mathrm{x}, \mathrm{y})<=255$.

\subsection{Color Image}

Color image is presented by a triple RGB (Red, Green, Blue). The value of color channel ranges from 0 to 255 . Set of 3 color channels will create $2^{24}$ colors $(256 * 256 * 256$ colors).

\subsection{Grayscale Image}

Grayscale images [5, 6] are the color images using RGB color system in which the Red, Green, Blue have the same light intensity. So, the grayscale image just need to use one light intensity to show each pixel. The gray level of grayscale image ranges from 0 to 255 .

\subsection{Histogram}

Histogram [5,7] is the chart that shows the frequency of occurrence of each gray level in an image.

- The horizontal axis $\mathrm{X}$ is the value of gray level.

- Y-axis is the number of pixels with gray levels corresponding to the image (frequency of occurrence).

Figure 1 illustrates the histogram of an image.

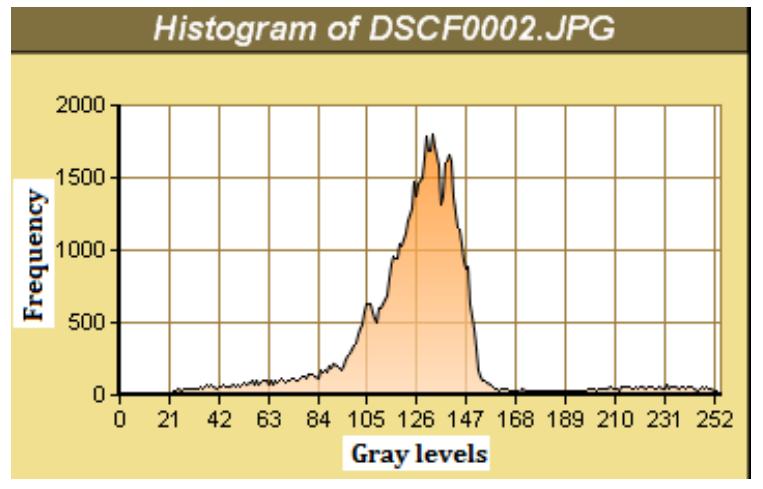

Fig 1. Histogram of image

\section{SYSTEM DESIGN}

\subsection{System Model}

The system model including 6 stages is presented in Figure 2 .

- The first stage: Color image obtained from the camera.

- The second stage: Converting color image into grayscale image.

- The third stage: Calculating histogram of grayscale image. 
- The fourth stage: Increasing the contrast of grayscale image based on histogram.

- The fifth stage: Determining the number of slices and calculating the derivative of slices.

- The sixth stage: Classifying the traffic density based on the derivative of slices.

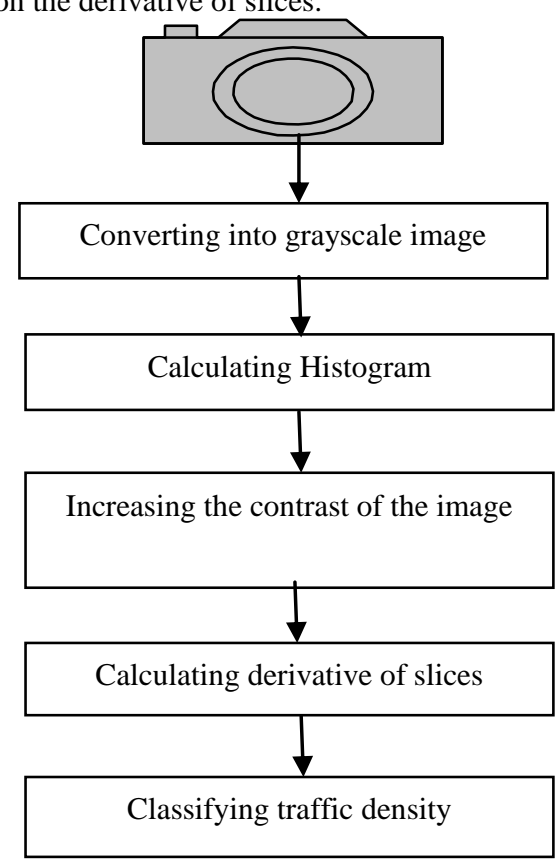

Fig 2. System Model

\subsection{Convert into Grayscale Image}

There are some techniques to convert color image into grayscale image such as lightness with the equation $(\max (\mathrm{R}$, $\mathrm{G}, \mathrm{B})+\min (\mathrm{R}, \mathrm{G}, \mathrm{B})) / 2$, luminosity with the equation $0.21 \mathrm{R}$ $+0.72 \mathrm{G}+0.07 \mathrm{~B}$. In this paper, the average method is chosen, so the value of pixel in grayscale image is calculated by (1).

$\mathrm{V}=(\mathrm{R}+\mathrm{G}+\mathrm{B}) / 3$

For example, one pixel of color image has $\mathrm{R}=140, \mathrm{G}=200$, $\mathrm{B}=98$, when converting into grayscale image, the value of pixel in grayscale image is $(140+200+98) / 3=146$.

\subsection{Calculating Histogram}

Calculating histogram of image is performed as follows:

- Building the pixel matrix of the grayscale image.

- From the pixel matrix of the grayscale image, building the frequency of pixel.

The figure 3 presents the procedure of calculating the histogram of the grayscale image.

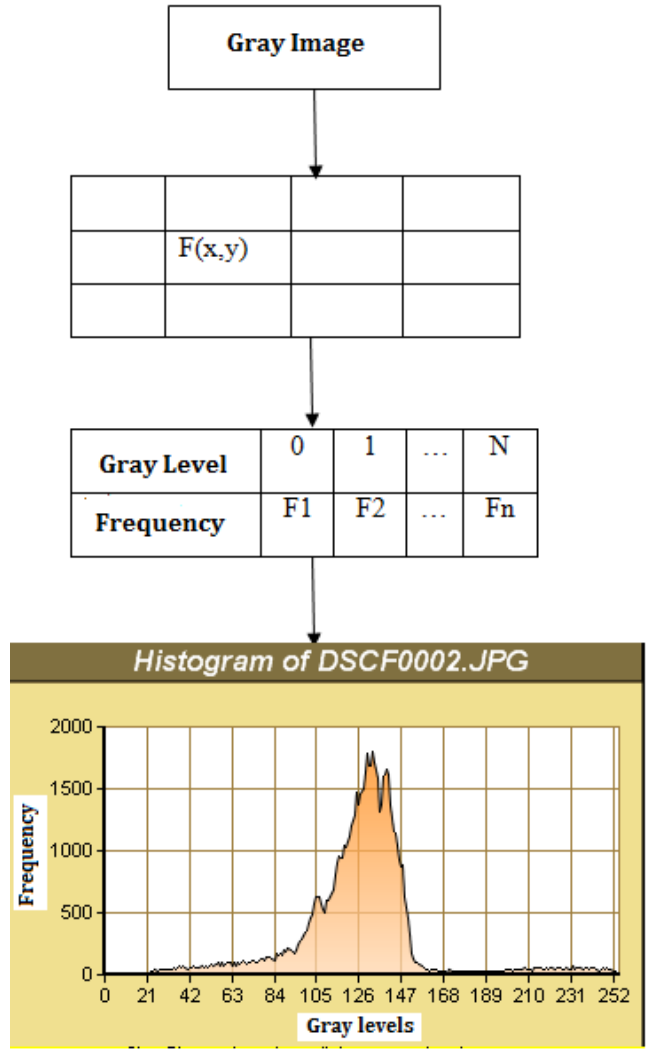

Fig 3. The procedure of calculating the histogram of image

\subsection{Increasing The Contrast Of Image}

Increasing the contrast of image is performed as follows:

- $\quad$ Normalizing histogram h(i) by (2).

$h_{n}(i)=\frac{h(i)}{N}, i \hat{\mathrm{I}}[0,255]$

- $\quad$ Calculating the probability density by (3).

$$
C(i)=\stackrel{\stackrel{\circ}{a}}{i} h_{n}(j), i \hat{\mathrm{I}}[0,255]
$$

- $\quad$ Find the greatest gray level (max).

- Transforming gray level by (4).

$\mathrm{f}(\mathrm{x}, \mathrm{y})=\mathrm{C}(\mathrm{x}, \mathrm{y}) * \max$

The figure 4, 5 and 6 illustrate color image, grayscale image and grayscale image with increasing contrast.

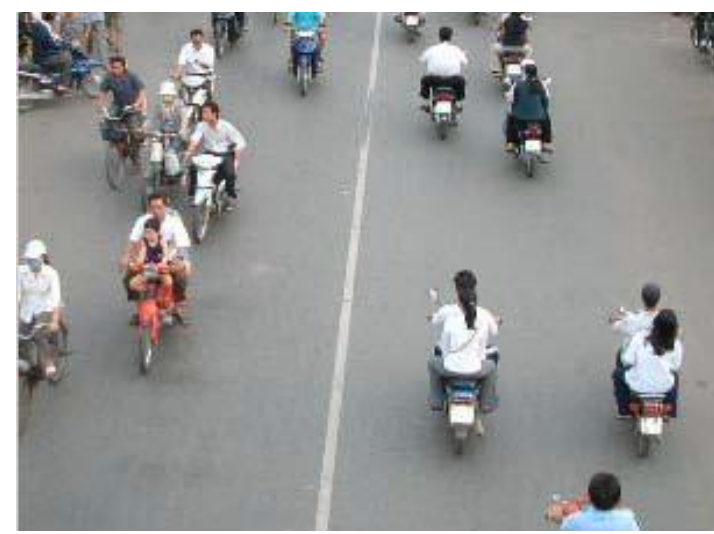

Fig 4. color image 


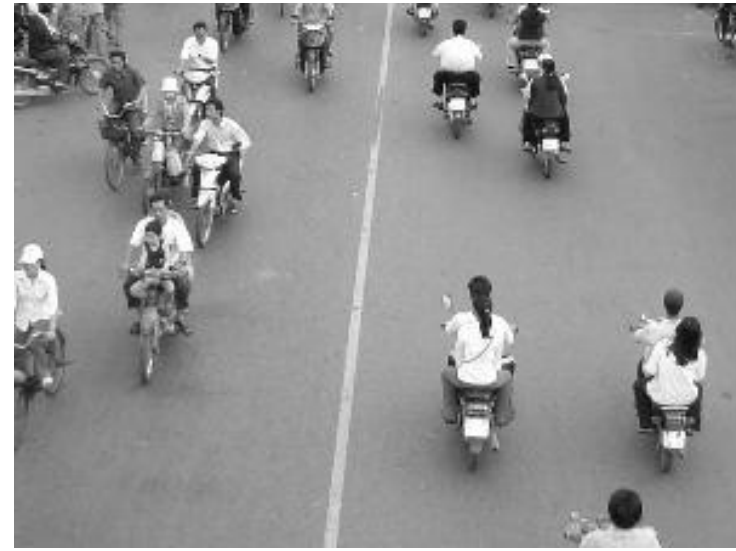

Fig 5. grayscale image

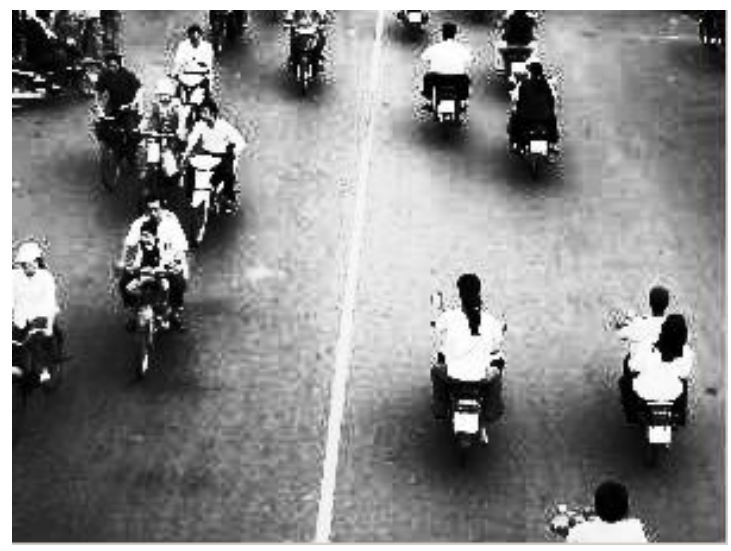

Fig 6. grayscale image with increasing contrast

\subsection{Calculating The Derivative of The Image}

This procedure is performed as follows:

- Considering horizontal slice $\mathrm{P}_{\mathrm{L}}\left(\mathrm{x}, \mathrm{y}_{0}\right)$.

- Calculating the derivative of $\mathrm{P}_{\mathrm{L}}\left(\mathrm{x}, \mathrm{y}_{\mathrm{o}}\right)$ by (5).

$$
P^{\prime}{ }_{L}\left(x, y_{0}\right)=\frac{\llbracket P_{L}\left(x, y_{0}\right)}{\llbracket x}
$$

- The derivative of $x_{i}$ is calculated by (6).

$$
f^{\prime}\left(x_{i}\right)=\lim _{\mathrm{D} x \mathbb{R} o} \frac{f\left(x_{i}+\mathrm{D} x\right)}{\mathrm{D} x}
$$

- In this problem, $\Delta \mathrm{x}$ is constant and its value is 1 . So, the derivative of $x_{i}$ is calculated by (7).

$$
f^{\prime}\left(x_{i}\right)=f\left(x_{i}+1\right)-f\left(x_{i}\right)
$$

\subsection{Classifying The Traffic Image Density}

- Determining threshold based on the characteristic of Vietnam traffic and the size of traffic objects.

- Comparing threshold with the derivative of slice to identify the traffic density.

\section{EXPERIMENT RESULTS}

\subsection{Dataset}

In this paper, 300 images were taken in Ho Chi Minh city, Vietnam on many different streets with different times. These images with the traffic density such as very crowded traffic, crowded traffic, normal traffic and sparse traffic are experimented in proposed technique.

\subsection{Experiment Procedure and Results}

A Software is designed by VB.NET programming language to experiment. The proposed technique is experimented with 10 slices, 15 slices , 20 slices and 25 slices. The experiment result is shown in table 1 . The best accuracy rate obtained $91 \%$.

Table 1. The Experiment result

\begin{tabular}{|c|c|c|}
\hline The number of slices & Accuracy rate & Time \\
\hline 10 & $76 \%$ & $27 \mathrm{~ms} /$ an image \\
\hline 15 & $83 \%$ & $36 \mathrm{~ms} /$ an image \\
\hline 20 & $91 \%$ & $47 \mathrm{~ms} /$ an image \\
\hline 25 & $91 \%$ & $55 \mathrm{~ms} /$ an image \\
\hline
\end{tabular}

The figure 7, 8, 9 and 10 present some experimental results with 10 slices.

\section{CONCLUSIONS}

In this paper, the traffic density classification technique based on horizontal slice algorithm and histogram is proposed. The technique is designed and experimented via VB.NET programming language with 300 images taken in $\mathrm{Ho}$ Chi Minh city, Vietnam on many different streets with different times. The results obtained with $91 \%$ accuracy. In the future, the proposed technique will be improved by the algorithm for determining the number of slices.

\section{REFERENCES}

[1] Ozkurt C, Camci F. Automatic traffic density estimation and vehicle classification for traffic surveillance systems using Neural Networks. Mathematical and Computational Applications. 2009; 14(3):187-96.

[2] C. Stuiz, T. A. Runkler, "Classification and Predicts of Road Traffic using Application Specific Fuzzy Clustering”, Fuzzy Systems, IEEE Transactions, pp. 297 308, 2002.

[3] Al Bovik (2000), Handbook of Image and Video Processing, Academic Press.

[4] Rafael C.Gonzalez, Richard E. Woods ( 1993), Digital Image Processing, Addison Wesley Pub.Comp.

[5] Luong Anh Tuan Nguyen, Huu Khuong Nguyen. Traffic Density Identification Based On Histogram. Journal of Transportation Science and Technology, ISSN: 18594263, Vol 15-05/2015, pp 23-27.

[6] Xiangyun Ye, Mohamed Cheriet, Senior Member, Ching Y. Suen (2001), Stroke-Model-Based Character Extraction from Gray-Level Document Images, IEEE.

[7] C. C. Sun. S. J. Ruan, M. C. Shie, T. W. Pai, "Dynamic Contrast Enhancement based on Histogram Specification," IEEE Transactions on Consumer Electronics, 51(4), pp.1300-1305, 2005. 


\section{APPENDIX}

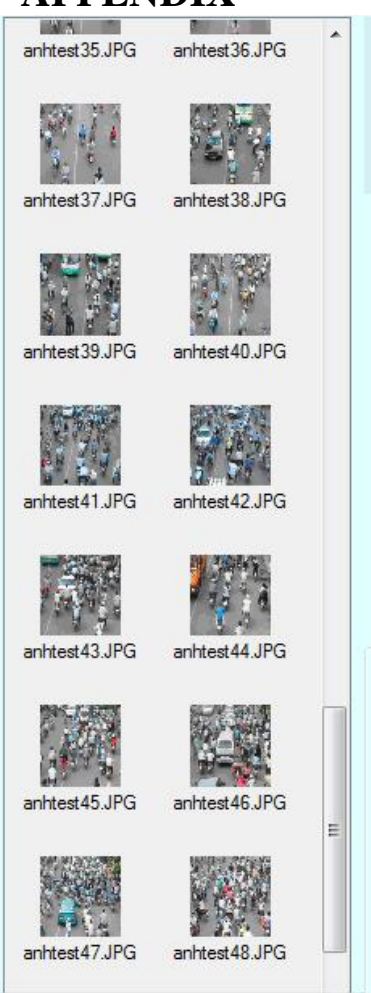

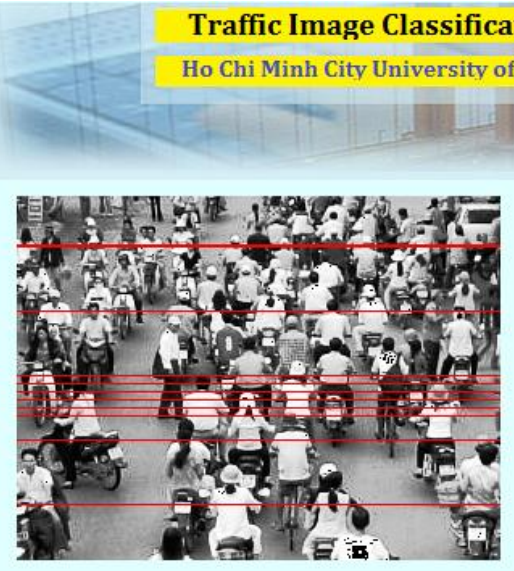

Result:

Image: anhtest48.JPG

Density: Very Crowded Traffic
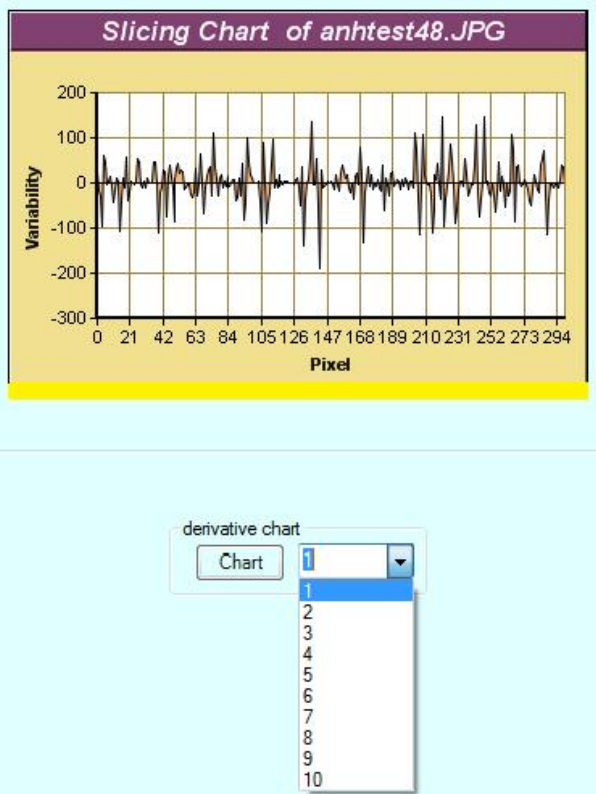

Fig 7. The result of classification "Very Crowded Traffic" with 10 slices
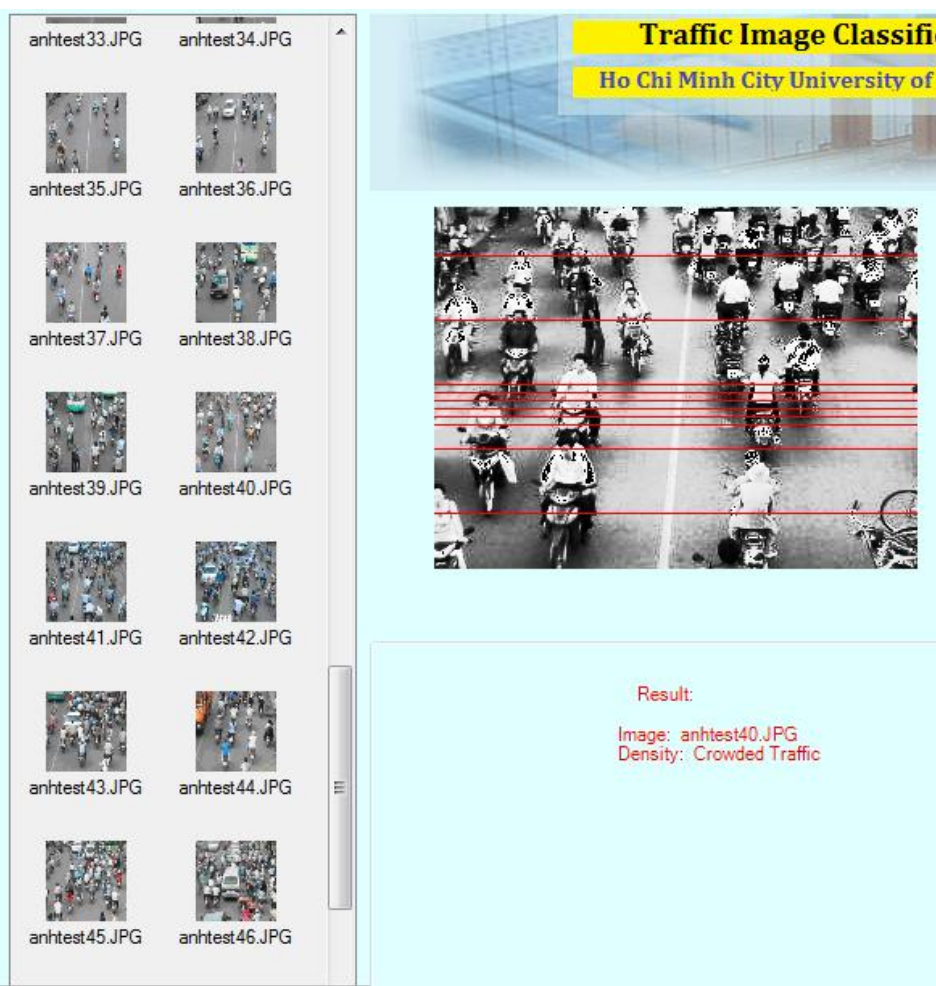

Traffic Image Classification Using Horizontal Slice Algorithm

Chi Minh City University of Transport

gge: anhtest40.JPG

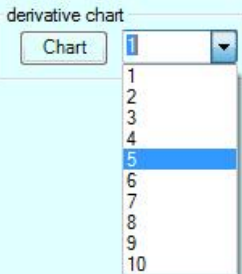

Fig 8. The result of classification "Crowded Traffic" with 10 slices 

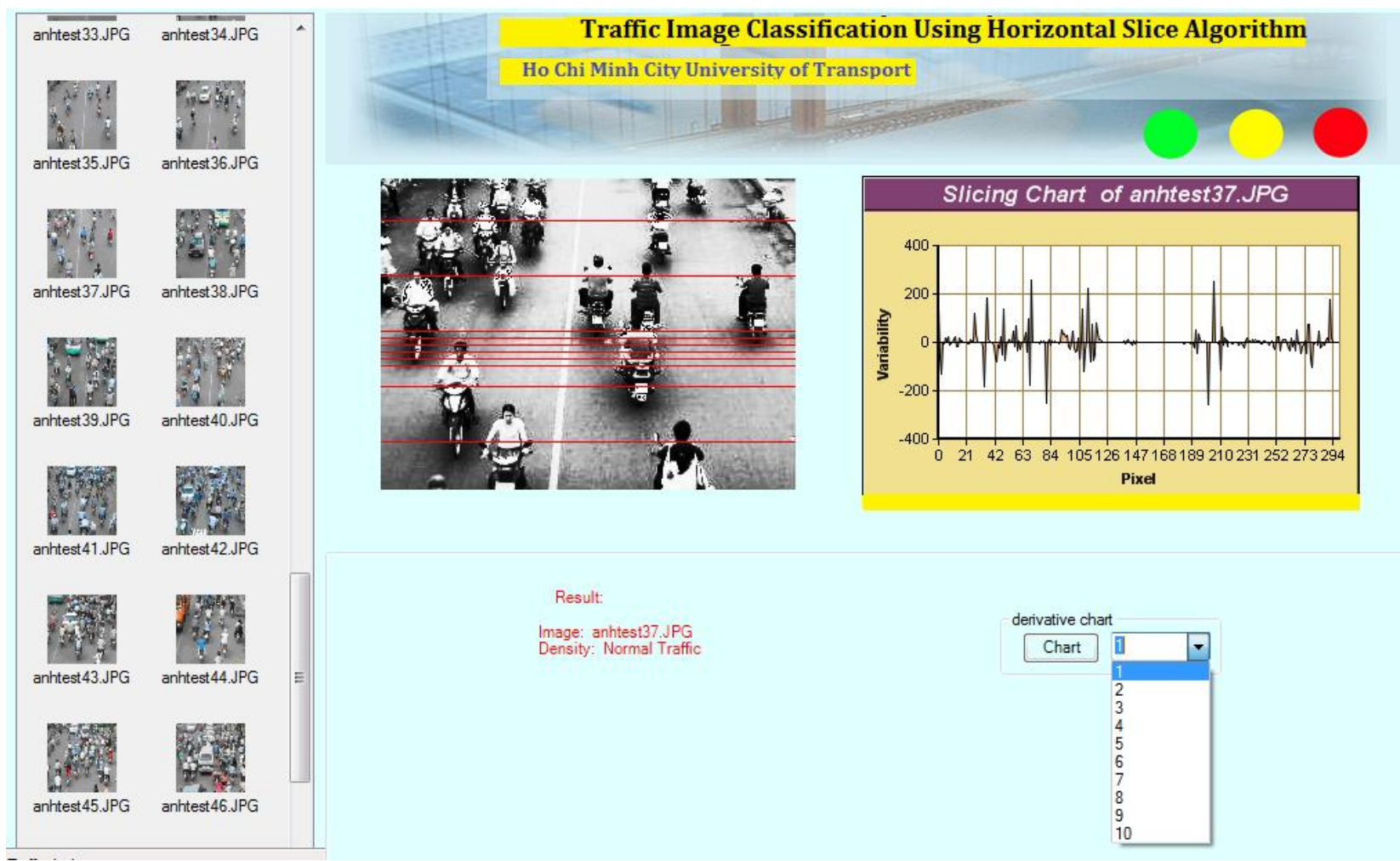

Fig 9. The result of classification "Normal Traffic" with 10 slices
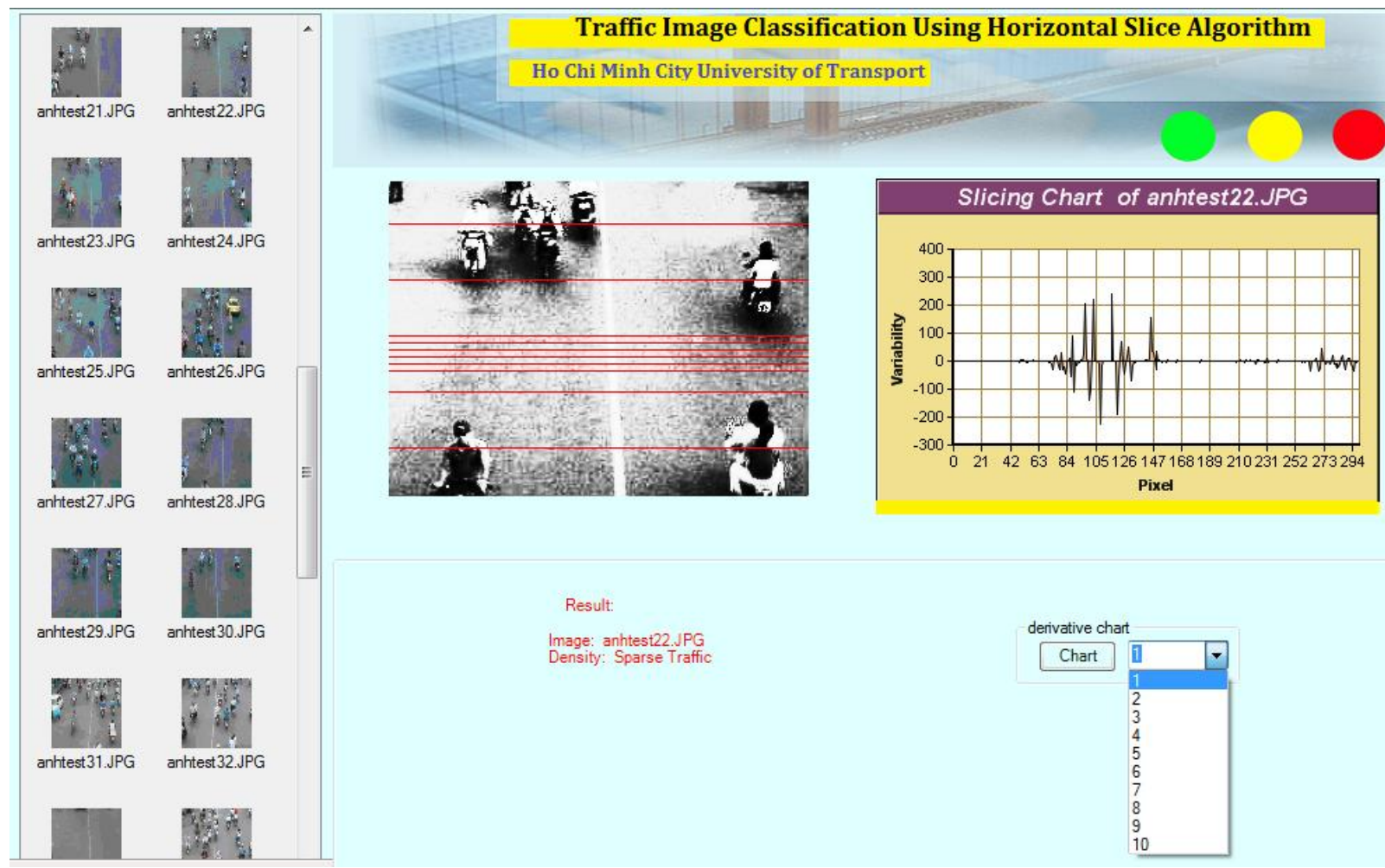

Fig 10. The result of classification "Sparse Traffic" with 10 slices 